\title{
A Lifting Result for Locally Pseudo-Convex Subspaces of $L_{0}$
}

by

\author{
Félix CABELLO SÁNCHEZ
}

Presented by Aleksander PEŁCZYŃSKI

Summary. It is shown that if $F$ is a topological vector space containing a complete, locally pseudo-convex subspace $E$ such that $F / E=L_{0}$ then $E$ is complemented in $F$ and so $F=E \oplus L_{0}$. This generalizes results by Kalton and Peck and Faber.

Introduction. Let $L_{0}$ denote the space of all (equivalence classes of) measurable functions on $[0,1]$ equipped with the topology of convergence in measure, $E$ a closed subspace of $L_{0}, \pi: L_{0} \rightarrow L_{0} / E$ the natural quotient map and $T: L_{0} \rightarrow L_{0} / E$ a (linear, continuous) operator. Under what conditions does $T$ lift to an operator $S: L_{0} \rightarrow L_{0}$ in the sense that the diagram

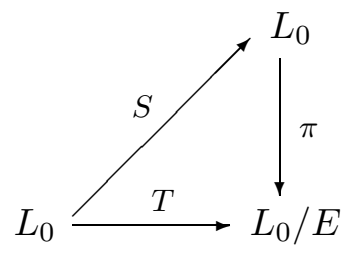

commutes? As far as I know this problem was raised by Pełczyński. Kalton and Peck [5, Theorem 3.6] proved that such an $S$ exists if $E$ is locally bounded (that is, a quasi-Banach space); see also [6, Theorem 6.4]. The same is true if $E$ is isomorphic to $\omega$, the space of all sequences, as follows from results of Peck and Starbird [7, Corollary]. The interesting work of Domański about the

2000 Mathematics Subject Classification: 46M18, 46A16, 46A22.

Key words and phrases: space of measurable functions, lifting, extension, pull-back, push-out.

Supported in part by DGICYT project MTM2004-02635. 
structure of extensions [2] contains alternative proofs of both resuls. Finally, Faber [3, Theorem 2.1] got the corresponding result for locally convex $E$.

In this short note we generalize the previous results to locally pseudoconvex subspaces of $L_{0}$. Actually, we will show that if $E$ is locally pseudoconvex and complete and $F$ is any topological vector space (TVS) containing it, then every operator $L_{0} \rightarrow F / E$ lifts to $F$. Thus, the fact that $E$ is a subspace of $L_{0}$ plays no rôle here. However we emphasize that there are locally pseudo-convex subspaces of $L_{0}$ that are neither locally convex nor locally bounded (nor even locally $p$-convex for any fixed $p$ ): $\prod_{n=1}^{\infty} L_{p(n)}$ is an example if the sequence $0<p(n) \leq 2$ converges to zero.

In contrast to Faber's proof (which is quite "hard" and depends on specific features of the locally convex subspaces of $L_{0}$ ) our result is obtained straightforwardly from the locally bounded case by means of the universal properties of three basic (and simple) homological constructions: pull-back, push-out and inverse limit.

Before going further we make some conventions. TVSs are assumed to be Hausdorff. Operator means linear and continuous map. If $E$ and $F$ are TVSs, then $L(E, F)$ denotes the space of all operators from $E$ to $F$. The identity on $E$ is written $1_{E}$.

Let us translate the problem into the language of extensions. An extension (of $G$ by $E$ ) is a short exact sequence of TVSs and relatively open operators

$$
0 \rightarrow E \stackrel{\imath}{\rightarrow} F \stackrel{\pi}{\rightarrow} G \rightarrow 0 .
$$

Less technically we can regard $F$ as a TVS containing $E$ as a subspace in such a way that $F / E$ is (isomorphic to) $G$. We say that (1) splits if there is $S \in L(G, F)$ such that $\pi \circ S=1_{G}$. And this happens if and only if there is $P \in L(F, E)$ such that $P \circ \imath=1_{E}$, that is, if $\imath E$ is a complemented subspace of $F$.

We now describe the algebraic constructions we shall use in the proof. Some verifications are left to the reader. They are really easy: just try or adapt the corresponding proof for (quasi-) Banach spaces in [4] or [1, Appendix].

1. The pull-back extension. Suppose we are given an extension (1) and an operator $L: H \rightarrow G$, where $H$ is a TVS. Then we can construct a commutative diagram

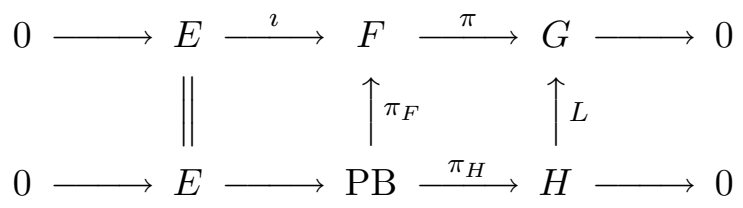


as follows: the pull-back space is $\mathrm{PB}=\{(f, h) \in F \times H: \pi f=L h\}$, with the relative product topology. The maps from $\mathrm{PB}$ are the restrictions of the projections. The map $E \rightarrow \mathrm{PB}$ is just $e \mapsto(\imath(e), 0)$. It is easily verified that the lower row in (2) is an extension which splits if and only if $L$ lifts to $F$. And this is so by the following universal property of the pull-back square: if $I$ is a TVS and $\alpha$ and $\beta$ are operators making the diagram

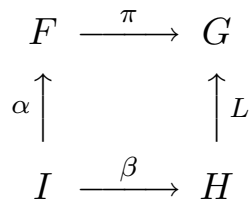

commutative, then there is a unique operator $\gamma: I \rightarrow \mathrm{PB}$ such that $\alpha=$ $\pi_{F} \circ \gamma$ and $\beta=\pi_{H} \circ \gamma$ (the converse is obvious).

Hence the following statements about a pair of TVSs $E$ and $H$ are equivalent:

- Whenever $F$ is a TVS containing $E$ every operator $H \rightarrow F / E$ lifts to $F$.

- Every extension $0 \rightarrow E \rightarrow I \rightarrow H \rightarrow 0$ splits.

Thus, the promised generalization of Faber's result is contained in the following:

FACT. Every extension of $L_{0}$ by a complete, locally pseudo-convex space splits.

Before going into the proof, let us describe

2. The push-out extension. The push-out construction is just the categorical dual of the pull-back. So assume we are given an extension (1) and an operator $T: E \rightarrow J$. The push-out of the operators $\imath$ and $T$ is the quotient space $\mathrm{PO}=(F \oplus J) / \Delta$, where $\Delta=\{-\imath(e) \oplus T(e): e \in E\}$. In our setting $\Delta$ is closed because $\imath$ has closed range. We have a commutative diagram

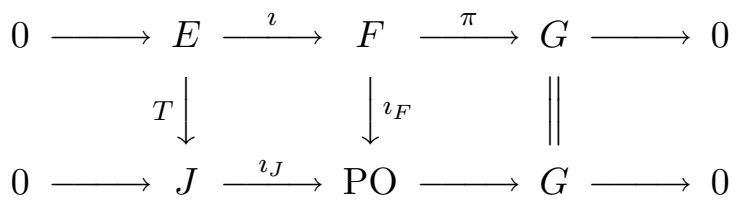

The arrows ending in $\mathrm{PO}$ are induced by the inclusions of $F$ and $J$ into their direct sum $F \oplus J$. The operator $\mathrm{PO} \rightarrow G$ sends $(f \oplus j)+\Delta$ to $\pi(f)$. This is clearly a quotient operator and it is easily seen that the lower sequence in (3) is an extension. Moreover this extension splits if and only if $T$ extends to $F$ (in the sense that there is $\tau \in L(F, J)$ such that $\tau \circ \imath=T$ ). Again, this is immediate from the universal property of the push-out construction: if $\alpha$ 
and $\beta$ are operators making the diagram

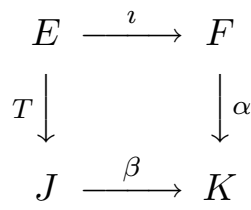

commutative, then there is a unique operator $\gamma: \mathrm{PO} \rightarrow K$ such that $\alpha=$ $\gamma \circ \imath_{F}$ and $\beta=\gamma \circ \imath_{J}$ (the converse is obvious).

3. The inverse limit. The topology of a locally pseudo-convex space $E$ can be obtained through a system of functions

$$
\varrho: E \rightarrow \mathbb{R}^{+} \quad(\varrho \in \Gamma),
$$

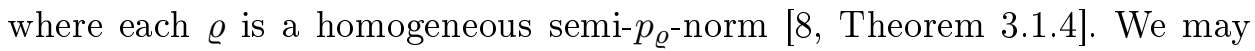
assume that given $\alpha, \beta \in \Gamma$ there is $\delta \in \Gamma$ such that $\delta \geq \alpha, \beta$ (in the pointwise sense). For $\varrho \in \Gamma$, let $E_{\varrho}$ denote the completion of $E / \operatorname{ker} \varrho$. This is clearly a $p_{\varrho}$-Banach space and we have an obvious operator $\pi_{\varrho}: E \rightarrow E_{\varrho}$. Moreover, if $\alpha \geq \beta$ the map $\pi_{\beta}$ factors through $E_{\alpha}$ and we have a further operator $\pi_{\beta}^{\alpha}: E_{\alpha} \rightarrow E_{\beta}$. It is clear that these form a projective system in the sense that for $\alpha \geq \beta \geq \gamma$ the map $E_{\alpha} \rightarrow E_{\gamma}$ coincides with the composition $E_{\alpha} \rightarrow E_{\beta} \rightarrow E_{\gamma}$.

Just as in the locally convex case, it is easily seen that if $E$ is complete, then it is isomorphic to the inverse (projective) limit of the system $\left\{E_{\gamma}: \gamma \in \Gamma\right\}$, that is, the space

$$
\operatorname{proj} E_{\gamma}=\left\{\left(e_{\gamma}\right) \in \prod E_{\gamma}: \pi_{\beta}^{\alpha}\left(e_{\alpha}\right)=e_{\beta} \text { for all } \alpha \geq \beta\right\}
$$

equipped with the relative product topology. We leave to the reader the verification that the map $e \in E \mapsto\left(\pi_{\gamma}(e)\right)_{\gamma} \in \prod E_{\gamma}$ defines an isomorphism between $E$ and $\operatorname{proj} E_{\gamma}$. Every operator $T: F \rightarrow E$ gives rise to a system of operators $T_{\gamma}: F \rightarrow E_{\gamma}$ (namely, $T_{\gamma}=\pi_{\gamma} \circ T$ ), compatible in the sense that for $\alpha \geq \beta$ we have $T_{\beta}=\pi_{\beta}^{\alpha} \circ T_{\alpha}$.

The universal property of the inverse limit states the converse: if $T_{\gamma}: F \rightarrow E_{\gamma}$ is a compatible system, then there is a unique operator $T: F \rightarrow E$ such that $T_{\gamma}=\pi_{\gamma} \circ T$.

Proof of the Fact. Let $E$ be a complete, locally pseudo-convex space. We show that every extension

$$
0 \rightarrow E \stackrel{\imath}{\rightarrow} F \stackrel{\pi}{\rightarrow} L_{0} \rightarrow 0
$$

splits. If $\varrho$ is a semi- $p$-norm on $E$ we can apply the push-out procedure to 
$\pi_{\varrho}$ and obtain the diagram

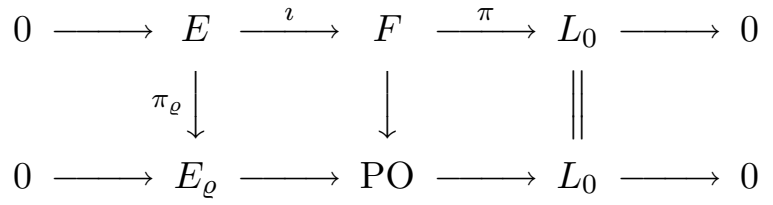

We know from [5] that the push-out extension splits and so there is $P_{\varrho}$ : $F \rightarrow E_{\varrho}$ such that $\pi_{\varrho}=\imath \circ P_{\varrho}$. In fact $P_{\varrho}$ is unique: for if $P: F \rightarrow E_{\varrho}$ is another extension of $\pi_{\varrho}$ we have $\left(P-P_{\varrho}\right) \circ \imath=0$ and so $P-P_{\varrho}$ factors through $L_{0}$. But the only operator from $L_{0}$ to a quasi-Banach space is zero, and so $P=P_{\varrho}$.

We claim that the system $\left(P_{\gamma}\right)_{\gamma} \in \Gamma$ defines an operator $P: F \rightarrow E$ such that $P \circ \imath=1_{E}$. Suppose $\alpha \geq \beta$ and let $P_{\alpha}$ and $P_{\beta}$ be as above. We have $\pi_{\alpha}=P_{\alpha} \circ \imath$ and $\pi_{\beta}=P_{\beta} \circ \imath$. Since $\pi_{\beta}=\pi_{\beta}^{\alpha} \circ \pi_{\alpha}$ we have $\pi_{\beta}=\pi_{\beta}^{\alpha} \circ P_{\alpha} \circ \imath$ and by the uniqueness of $P_{\beta}$ we see that $P_{\beta}=\pi_{\beta}^{\alpha} \circ P_{\alpha}$. This implies that there is an operator $P: F \rightarrow E$ such that $P_{\gamma}=\pi_{\gamma} \circ P$ for all $\gamma \in \Gamma$, which clearly implies that $P \circ \imath=1_{E}$ and completes the proof.

CONCLUDING REMARKS. Of course, the result just proved implies that if $E$ and $F$ are locally pseudo-convex (closed) subspaces of $L_{0}$ such that $L_{0} / E$ and $L_{0} / F$ are isomorphic, then there is an automorphism of $L_{0}$ mapping $E$ onto $F$.

Let us say that a TVS $G$ has $L_{0}$-structure if for every neighborhood of the origin $U$ there is a topological decomposition $G=G_{1} \oplus \cdots \oplus G_{k}$ with $G_{i} \subset U$ for $1 \leq i \leq k$. By [5, Theorem 3.6] (or [2, Proposition 4.3]) every extension of such a $G$ by any quasi-Banach space splits. Moreover, there is no nonzero operator from $G$ into any quasi-Banach space, and so the above proof shows that every extension of $G$ by a complete, locally pseudo-convex space splits. The condition on the operators cannot be removed: indeed, $\omega$ has "almost" $L_{0}$-structure: if $U$ is a neighborhood of zero, we can write $\omega=F \oplus G$, where $F$ is finite-dimensional and $G \subset U$. It follows that every extension of $\omega$ by a quasi-Banach space splits. However, it is shown in [2] (see the counterexamples on p. 166) that there exists an extension $0 \rightarrow E \rightarrow F \rightarrow \omega \rightarrow 0$ in which $F$ (and so $E$ ) is a Fréchet space that does not split.

The completeness hypothesis is also necessary in the Fact. Indeed, assume $E$ is locally pseudo-convex but not complete and let $\widehat{E}$ be its completion (clearly locally pseudo-convex). Consider the extension $0 \rightarrow E \rightarrow \widehat{E} \rightarrow$ $\widehat{E} / E \rightarrow 0$, where the quotient space carries the trivial topology (the only open sets are the empty one and the whole space). Now, let $T: L_{0} \rightarrow \widehat{E} / E$ be any nonzero linear map; this is clearly an operator that cannot be lifted to $\widehat{E}$ since $L\left(L_{0}, \widehat{E}\right)=0$. Thus, the lower extension in the pull-back diagram 
(which can be defined as in the Hausdorff case and has the same properties)

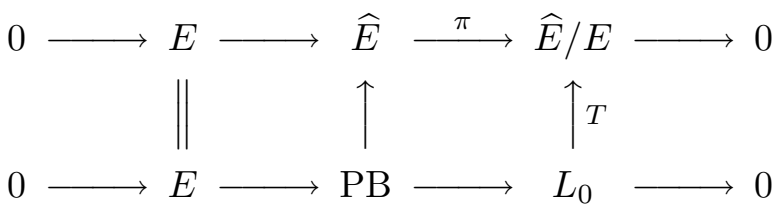

does not split. This is clearly a rewording of [2, "only if" part of Proposition $4.3(\mathrm{c})]$.

We close with the following

Problem. Does every extension $0 \rightarrow L_{0} \rightarrow F \rightarrow L_{0} \rightarrow 0$ split?

Acknowledgements. I thank Jesús M. F. Castillo for explaining to me-again - how a projective limit works (and many other things), and Javier Cabello Sánchez for reading a preliminary $\mathrm{LT}_{\mathrm{E}} \mathrm{X}$-script of this note.

\section{References}

[1] F. Cabello Sánchez and J. M. F. Castillo, The long homology sequence of quasiBanach spaces, with applications, Positivity 8 (2004), 379-394.

[2] P. Domański, On the splitting of twisted sums and the three space problem for local convexity, Studia Math. 82 (1985), 155-189.

[3] R. G. Faber, A lifting result for locally convex subspaces of $L_{0}$, ibid. 115 (1995), $73-85$.

[4] W. B. Johnson, Extensions of $c_{0}$, Positivity 1 (1997), 55-74.

[5] N. J. Kalton and N. T. Peck, Quotients of $L_{p}$ for $0 \leq p<1$, Studia Math. 64 (1979), $65-75$.

[6] N. J. Kalton, N. T. Peck, and J. W. Roberts, An F-Space Sampler, London Math. Soc. Lecture Note Ser. 89, Cambridge Univ. Press, 1984.

[7] N. T. Peck and T. Starbird, $L_{0}$ is $\omega$-transitive, Proc. Amer. Math. Soc. 83 (1981), 700-704.

[8] S. Rolewicz, Metric Linear Spaces, Reidel and PWN, Dordrecht-Warszawa, 1985.

Félix Cabello Sánchez

Departamento de Matemáticas

Universidad de Extremadura

Avenida de Elvas

06071 Badajoz, Spain

E-mail: fcabello@unex.es

Web: http://kolmogorov.unex.es / ${ }^{\sim}$ fabello

Received March 15, 2006;

received in final form October 10, 2006 